\title{
CHINESE HERBAL MEDICINE: A SAFE ALTERNATIVE THERAPY FOR URINARY TRACT INFECTION IN PATIENTS WITH RENAL INSUFFICIENCY
}

\section{YanQing Tong, Yan Leng ${ }^{2}$ and JingLi Bai ${ }^{2}$}

The First Affilliated Hospital to Changchun University of Chinese Medicine, Department of Nephrology; Gongnong Road, No 1478, Changchun city, Jilin province, China, 130021, ${ }^{2}$ Department of Nephrology, The First Affilliated Hospital to Changchun University of Chinese Medicine, Changchun city, Jilin province, China.

*Email: tyq1229@yahoo.com.cn

\begin{abstract}
We have used reduced doses of Chinese herbs for estimation of urinary tract infections (UTIs) patients with stable impairment of renal function. A total of 33 adult female patients with moderately impaired renal function and symptomatic UTIs were included in this study. Urine cultures were carried out. Patients were monitored clinically and with various laboratory tests. Chinese herbal concoction divided by milligrams of creatinine per $100 \mathrm{ml}$ were orally administrated for ten days. Three patients were excluded from final analysis. Most of the patients responded symptomatically to treatment. Chinese herbs eradicated the primary pathogen in $68.7 \%$ of the patients at the day 10 of treatment. Two patients relapsed (one had abbreviated courses of therapy) 6 to 8 days posttreatment. Organisms which recurred included Escherichia coli and Pseudomonas aeruginosa. Bacterial reinfections occurred 5 to 8 days posttreatment in four patients. Adverse reactions observed among the 30 patients were rare. Nausea (6.67\%) and mild elevation of hepatic enzymes (3.33\%) were probably drug related. Nausea disappeared when the therapy ended. Elevated hepatic enzymes resumed at the 2-week follow-up. Two patients demonstrated slight increases in serum creatinine on day 10 of treatment. One patient had a $12.5 \%$ elevation over baseline and the other had a $13.0 \%$ elevation. Serum creatinine values had improved in these two patients at 4-week follow-up. Chinese herbal medicine was effective and safe in the treatment of UTIs with renal insufficiency.
\end{abstract}

Key words: Chinese herbs; Urinary tract infection; Renal insufficiency; Dose adjustment.

\section{Introduction}

Complicated urinary tract infections (UTIs) may occur in all age groups and are frequently associated with either structural or functional urinary tract abnormalities. Examples include foreign bodies such as calculi (stones), indwelling catheters or other drainage devices, obstruction, immunosuppression, and renal failure (Lichtenberger et al., 2008). The management of UTIs in patients with chronic renal insufficiency has attracted little attention. An in-depth literature review identified only one publication that addressed the subject (Bennett and Craven, 1976). Many UTIs-controlled studies excluded patients with impaired renal function. UTIs in these patients seems not to be a problem that is sufficient to generate academic interest.

Herbal remedies are herbs, herbal materials, herbal preparations and finished herbal products, that contain as active ingredients parts of plants, or plant materials, or combinations thereof used to treat a multitude of ailments throughout the world (WHO, 2002). In our previous study, some Chinese herbs were shown to be clinically effective and relatively free from toxic side effects when given orally to patients of UTIs caused by fluoroquinolone-resistant strains. The patients http://dx.doi.org/10.4314/ajtcam.v9i2.13 
participating in the study had normal renal function (Tong et al., 2011b).

However, every drug is a potential poison. Thus some herbal remedies are toxic especially when used over a period. The maintenance dose for Chinese herbal drugs in continuous drug administration is inversely proportional to the half-life of the drug at reduced renal function. It is postulated that dividing the normal daily dosage by the patient's serum creatinine (in milligrams per $100 \mathrm{ml}$ ) will result in effective serum and urine concentrations in the treatment of UTIs and not cause an accumulation of drug in the patient's serum. In the present study, we have used reduced doses of these Chinese herbs for estimation of serious UTIs patients with stable impairment of renal function, based solely on pretreatment serum creatinine.

\section{Materials and methods}

Study protocol was approved by the First Affilliated Hospital to Changchun University of Chinese Medicine. Protocol summaries were reviewed by the participants, and written, informed consent was obtained on the day of the study after a detailed explanation of the study purpose and methods. A total of 33 adult female patients with moderately impaired renal function (serum creatinine, 1.5 to $2.5 \mathrm{mg} / 100 \mathrm{ml}$ ) and symptomatic UTIs (2 with bacteremia) were included in this study from April 2008 to September 2009. The patients were generally older (36 to 75 years old; mean, 58 years) with significant underlying diseases. Among group, 21 had neurogenic bladders, vesicoureteral reflux, calculi, or obstructive lesions, 11 were diabetes, and 8 required intermittent or indwelling urinary catheters; and 25 required hospitalization for their infections or were hospitalized for other physical illnesses when treatment was initiated.

The diagnosis of UTIs was based on the presence of signs and symptoms suggestive of UTIs, the presence of pyuria and bacteriuria. Urine cultures with colony counts $\geq 10^{5} \mathrm{CFU}$ were carried out on midstream clean-catch specimens before treatment. The urine cultures were repeated at 5 days, 10 days, and 1 week after the treatment. All urine samples were plated initially on blood agar plates and were quantitated by pour plates or calibrated wire loops. Cultures were observed for growth for 48 hrs. All bacterial isolates were identified by standard procedures (Edwards and Ewing,1955).

Patients were monitored clinically and with various laboratory tests to determine the incidences of adverse reactions. Blood urea nitrogen, serum creatinine, creatinine clearance, hemoglobin, hematocrit, total leukocyte count with differential count, alkaline phosphatase, lactate dehydrogenase, serum glutamic oxaloacetic transaminase, and serum bilirubin were determined in all patients before and after the study. The criterion for nephrotoxicity was a $20 \%$ elevation in creatinine over baseline.

All the patients were orally administrated Chinese herbal concoction. The prescription was as follow: Tong Cao (Stachyurus himalaicus Hook.f.et Thoms) 20g, Hua Shi (Talcum) 15g, Chi Shao (Paeonia lactiflora Pall.) 15g, Hui Xiang (Foeniculum vulgare Mill.) 15g, Guan Gui (Cinnamomum cassia Presl) 15g, Li Zhihe (Litchi chinensis Sonn) 15g, Tian Kuizi (Semiaquilegia adoxoides (DC.) makino) 15g, Zihua Diding (Viola ycdoensis Mak.) 20g, Ju Mai (Dianthus superbus L.) 15g, Ma Chixian (Portulaca oleracea L) 50g, Pu Gongying (Herba taraxaci) 30g. The concoction was prepared by mixing the crude drugs in $800 \mathrm{ml}$ water, getting $200 \mathrm{ml}$ liquor after the drugs were decocted in $800 \mathrm{ml}$ water $\left(100{ }^{0} \mathrm{C}\right.$ for 30 mins twice). After cooling, the concoction was stored at $18-24{ }^{0} \mathrm{C}$, humidity $55 \%-70 \%$. The concoction was orally administrated at $200 \mathrm{ml} /$ day divided by milligrams of creatinine per $100 \mathrm{ml}$ for ten days.

The therapeutic results were assessed according to the urine cultures. The following standards were used: cure, negative culture at 1 week after treatment; persistence, $\geq 10^{5}$ colonies of the original organism per ml during treatment; relapse, negative culture during therapy and $\geq 10^{5}$ colonies of the original organism per $\mathrm{ml}$ at follow-up; reinfection, $\geq 10^{5}$ colonies of an organism different from the original organism per $\mathrm{ml}$ at follow-up; and superinfection, $\geq 10^{5}$ colonies of an organism different from the original organism per $\mathrm{ml}$ during treatment. This assessment was based on routine bacteriological identification of the microorganisms since serotyping was not done. 


\section{Results}

Among the 33 patients entered into the study, 3 were excluded from final analysis for the following reasons: inadequate follow-up for 1 patient, and administration of antibiotics or other protocol violations in 2 patients.

The etiologic agents isolated from urine cultures before treatment were (with number of strains in parentheses): Escherichia coli (21), Pseudomonas aeruginosa (5), Klebsiella pneumoniae (3), Proteus mirabilis (2), and one each for Staphylococcus epidermidis, Staphylococcus aureus, Proteus morganii, and Providencia rettgeri. Two patients were infected with two organisms.

Most of the patients responded symptomatically to treatment. Bacteriologic outcomes are shown in Table 1. 50\%-100\% pathogens (except Proteus morganii) were eradicated by days 5 to 10 of treatment. Chinese herbs eradicated the primary pathogen in $68.7 \%$ of the patients at the day 10 of treatment. Two patients relapsed (one had abbreviated courses of therapy) 6 to 8 days posttreatment. Organisms which recurred included Escherichia coli and Pseudomonas aeruginosa. One patient grew Acinetobacter iwoffii on the last day of treatment. Bacterial reinfections occurred 5 to 8 days posttreatment in four patients. In all cases of relapse, and reinfection, patients had neurologic bladders, or urinary catheters.

Table 1: Bacteriologic response subclassified by etiology

\begin{tabular}{|c|c|c|c|c|c|c|c|}
\hline \multirow{3}{*}{ Organism } & \multirow{3}{*}{$\begin{array}{l}\text { No. of } \\
\text { patients }\end{array}$} & \multicolumn{6}{|c|}{$\%$ of patients } \\
\hline & & \multicolumn{2}{|c|}{ Day 5 of treatment } & \multicolumn{2}{|c|}{ Day 10 of treatment } & \multicolumn{2}{|c|}{ 1-week follow-up } \\
\hline & & Eradication & Persistence & Eradication & Persistence & Eradication & Persistence \\
\hline Escherichia coli & 20 & 45.0 & 55.0 & 70.0 & 30.0 & 65.0 & 35.0 \\
\hline Pseudomonas aeruginosa & 5 & 40.0 & 60.0 & 80.0 & 20.0 & 60.0 & 40.0 \\
\hline Klebsiella pneumoniae & 2 & 0 & 100.0 & 50.0 & 50.0 & 50.0 & 50.0 \\
\hline Proteus mirabilis & 2 & 50.0 & 50.0 & 50.0 & 50.0 & 50.0 & 50.0 \\
\hline Staphylococcus epidermidis & 1 & 0 & 100.0 & 100.0 & 0 & 100.0 & 0 \\
\hline Staphylococcus aureus & 1 & 0 & 100.0 & 100.0 & 0 & 100.0 & 0 \\
\hline Proteus morganii & 1 & 0 & 100.0 & 0 & 100.0 & 0 & 100.0 \\
\hline
\end{tabular}

Adverse reactions observed among the 30 patients were rare. Nausea (6.67\%) and mild elevation of hepatic enzymes $(3.33 \%)$ were probably drug related. Nausea disapeared when the therapy ended. Elevated hepatic enzymes resumed at the 2-week follow-up. Complaints of nervousness, blurred vision, and aching arms were not associated with objective findings and were of equivocal significance.

Two patients demonstrated slight increases in serum creatinine on day 10 of treatment. No. 1 patient had a $12.5 \%$ elevation over baseline and no. 2 had a 13.0\% elevation (Table 2). Increased renal impairment was attributed to possible nephrotoxic reaction to herbal remedy. The changes in serum creatinine and blood urea nitrogen are depicted in Table 2. One patient (no. 2 in Table 2) received small doses of diuretics. Dialysis was not attempted due to the general clinical condition and the underlying malignant disease of the patients. Serum creatinine values had improved in these two patients at 4-week follow-up. 
Table 2: Serum creatinine and blood urea nitrogen values in two patients

\begin{tabular}{|c|c|c|c|c|c|c|c|}
\hline \multirow[b]{2}{*}{$\begin{array}{c}\text { Patient } \\
\text { No. }\end{array}$} & \multirow{2}{*}{$\begin{array}{c}\text { Creatinine } \\
\text { Clearance } \\
\text { Pretreatment } \\
(\mathrm{ml} / \mathrm{min})\end{array}$} & \multicolumn{3}{|c|}{ Serum creatinine (mg/100ml) } & \multicolumn{3}{|c|}{ Blood urea nitrogen $(\mathrm{mg} / 100 \mathrm{ml})$} \\
\hline & & Pretreatment & $\begin{array}{l}\text { Last day of } \\
\text { treatment }\end{array}$ & $\begin{array}{c}\text { 4-week } \\
\text { follow-up }\end{array}$ & Pretreatment & $\begin{array}{l}\text { Last day } \\
\text { of } \\
\text { treatment }\end{array}$ & $\begin{array}{l}\text { 4-week } \\
\text { follow-up }\end{array}$ \\
\hline 1 & 48 & 1.6 & 1.8 & 1.7 & 37 & 40 & 38 \\
\hline 2 & 22 & 2.3 & 2.6 & 2.4 & 34 & 46 & 40 \\
\hline $\begin{array}{c}\text { Increase from } \\
\text { pretreatment } \\
\text { Values }\left(\text { Mean } \pm \mathrm{SD}^{a} \text { ) }\right.\end{array}$ & & & $0.25 \pm 0.05$ & 0 & & $\begin{array}{c}7.50 \pm \\
4.50\end{array}$ & $3.50 \pm 2.50$ \\
\hline
\end{tabular}

${ }^{a} \mathrm{SD}$, Standard deviation

\section{Discussion}

In UTIs, the clearance is abnormal in about half of the cases on the affected side. Contralateral compensation and intrarenal compensation mechanisms tend to occur. In addition to these compensation mechanisms, hyperfiltration is present in many cases of UTIs with intact or unilaterally damaged kidneys (Francisca et al., 1999). So the management of UTIs in patients with renal insufficiency is crucial to prevent renal function deterioration. Some Chinese herbs in the study have been reported with antibacterial activity (Tong and Li, 2006). Our early clinical study indicated that oral Chinese herbal concoction is effective in UTIs caused by fluoroquinolone-resistant organisms. Another previous research show that anti-adhesion is one mode of action for Chinese herbs used against UTIs pathogens (Tong et al., 2011b). These properties have prompted their compound prescription uses in the management of UTIs.

In the present study of patients with serious UTIs, bacteriuria was eradicated in $68.7 \%$ of 30 patients at day 10 of treatment. The main factor governing a poor outcome of treatment for these patients seems to be impaired renal function, neurogenic bladders, structural abnormalities of the genitourinary tract, or urinary catheters. All two patients who relapsed 6 to 8 days posttreatment had underlying urologic diseases requiring catheterization. Indwelling urinary catheters further favor the colonization of uropathogens by providing a surface for the attachment of host cell binding receptors that are recognized by bacterial adhesins. Upon insertion, urinary catheters may damage the protective uroepithelial mucosa, which leads to the exposure of new binding sites for bacterial adhesins (Garibaldi et al., 1980).

More prolonged treatment in these patients may have been more effective in eradicating their infections. One patient who became colonized with Acinetobacter iwoffii on the last day of treatment and the four patients who became reinfected with new organisms 5 to 8 days posttreatment, the lack of permanent cure was undoubtedly host related rather than drug related.

The safety of herbal remedy determines the well being of the patient taking the medication. Oral Chinese herbal concoction is well tolerated. Nausea and mild elevation of hepatic enzymes were observed. These adverse reactions disappeared after discontinuation of the medication. Although we could not exclude Chinese herbs as a contributory factor, these events were probably caused by unrelated underlying problems.

The renal function profile is a major concern in drug therapy. Special attention should be directed to patients with diminished renal function. Careful monitoring of creatinine clearance are indicated. Our definition of nephrotoxic reaction is sensitive. It should be emphasized that interpretation of changes in renal function is difficult in patients with uronephrological 
disorders. As observed by us, the nephrotoxic reaction to these Chinese herbs is reversible, which is probably due to applying the formula for dose adjustment proposed here. Further studies with larger populations are needed to show if the low nephrotoxic potential accounted for can be reproduced.

In short, the management of UTIs in patients with renal insufficiency requires reduction of dosage to avoid high serum concentrations and the concomitant increased risk for concentration-dependent adverse drug events. The appropriate duration of Chinese herbal therapy in patients with renal insufficiency is unknown. Duration studies are needed in the future. The design of our study has some shortcomings. A randomized-controlled double-blind trial would have been ideal but was not deemed feasible in this study given the oral route of administration for Chinese herbal concoction. Obviously this initial limited therapeutic trial must be extended to a larger group of patients and include long-term follow-up evaluations before a definite conclusion can be drawn.

In spite of these limitations, we considered Chinese herbs to be effective and safe in the treatment of UTIs with stable impairment of renal function. Increases in dosage intervals or shorter periods of treatment should theoretically result in less toxicity, but probably also reduce effectiveness. Prospective studies comparing dosage regimens with regard to these factors are required.

\section{Acknowledgments}

The help of ZhengPing Qu and Yue Jing, nurses in Department Nephrology, who took care of the patients, was greatly appreciated. We acknowledge the help of FangMing Gu for artwork. This study was funded by the Jilin Provincial Science \& Technology Department.

\section{References}

1. Bennett WM, Craven R. (1976). Urinary tract infections in patients with severe renal disease. JAMA. 236: 945-948.

2. .Edwards PR, Ewing, WH. (1955). Identification of Enterobacteriaceae. Burgess Publishing Company, Minneapolis, Minn, 25-27.

3. Francisca A, Hamphrey RH, Marianne T, Amy P. Overall and Single-Kidney Clearance in Children with Urinary Tract Infection and Damaged Kidneys. J Nucl Med, 1999, 40: 52-55.

4. Garibaldi RA, Burke JP, Britt MR, Miller MA, Smith CB. (1980). Meatal colonization and catheter- associated bacteriuria. N Engl J Med, 303: 316-318.

5. Lichtenberger P, Hooton TM. (2008). Complicated urinary tract infections. Curr Infect Dis Rep, 10: 499-504.

6. WHO. (2002). Traditional Medicine; Growing Needs and Potential, WHO Policy Perspectives on Medicines. World Health Organization, Geneva, 1-6.

7. Yanqing Tong, Hui Li. (2006). In-vitro Antibacterial Activity of the Combination of Herba Violae and Herba Taraxaci in Different Proportions. J of Guangzhou University of TCM, 23: 487-492.

8. YanQing Tong, Qiucheng Wu, DongKai Zhao, Yanru Liu, Mingming Cao, Liping Zhang, Shiming Zeng. (2011a). Effects of Chinese herbs on the hemagglutination and adhesion of Escherichia Coli strain in vitro. Afr J Tradit Complement Altern Med, 8:82-87.

9. Yanqing Tong, Yue Jing, Dongkai Zhao, Liping Zhang, Shiming Zeng. (2011b). Fluoroquinolone-resistant uncomplicated urinary tract infections, Chinese herbal medicine may provide help. Afr J Tradit Complement Altern Med, 8(S): 108-114. 